\title{
ESTUDO DO CONSUMO DE PLANTAS MEDICINAIS OBTIDAS EM FEIRAS LIVRES DE SÃO LUÍS, PARA O TRATAMENTO DE DOENÇAS CRÔNICAS.
}

\section{STUDY OF THE CONSUMPTION OF MEDICINAL PLANTS OBTAINED IN FREE FAIRS OF SÃO LUÍS, FOR THE TREATMENT OF CHRONIC DISEASES.}

\author{
Wellison Amorim Pereira ${ }^{1}$; Nágila Layane Rabelo Cutrim² ${ }^{2}$ Tatiane Santos Reis ${ }^{3}$; \\ Patrícia Heleny Pereira de Almeida ${ }^{4}$; Elizangela Araújo Pestana Motta ${ }^{5}$
}

RESUMO: As doenças crônicas se estabelecem como um problema de saúde pública internacional, em especial no Brasil. Plantas medicinais têm sido largamente usadas para tratar tais doenças, porém, sem os devidos cuidados, seu uso pode causar intoxicação e até a morte. Trata-se de um estudo de campo, descritivo e quantitativo, que objetivou investigar, listar e realizar a identificação botânica das plantas medicinais mais utilizadas pelos moradores de São Luís, para o tratamento da Hipertensão, Diabetes, Asma e Obesidade. Os dados das indicações foram obtidos após visitas às principais feiras da cidade de São Luís. Após o levantamento das sete plantas mais indicadas para cada patologia, as mesmas foram compradas e armazenadas na forma de exsicatas para sua póstuma identificação botânica, para saber se estas correspondiam ao que era comercializado pelos feirantes. Observou-se que apenas a parte que a qual era atribuída à ação farmacológica, era vendida, fator que impossibilitou a identificação botânica, levantando à discussão sobre os perigos da venda destes produtos. Foi feita ampla busca em periódicos científicos com os nomes populares das plantas informadas pelos feirantes, com o intuito de verificar sua eficácia no tratamento das doenças, sendo verificado que todas elas apresentavam eficácia comprovada. O presente estudo proveu maiores informações sobre os hábitos de consumo de plantas medicinais dos moradores de São Luís, assim como seus riscos e benefícios.

PALAVRAS-CHAVE: Plantas Medicinais; Doenças Crônicas; Fitoterapia.

\footnotetext{
1 Biomédico. Mestrando em Biologia Parasitária (Universidade CEUMA/FAPEMA). E-mail: wellison.amorim@hotmail.com

2 Biomédica pela Faculdade Estácio de São Luís. E-mail: ncutrim@live.com

${ }^{3}$ Biomédica pela Faculdade Estácio de São Luís . E-mail: tatianesantosreis@ outlook.com

${ }^{4}$ Biomédica pela Faculdade Estácio de São Luís . E-mail:patriciaheleny @ hotmail.com

${ }^{5}$ Farmacêutica, Doutoranda em Ciências da Saúde (UFMA); Mestre em Química (UFRN), Docente Faculdade Estácio de São Luís, Membro do CEP da Universidade CEUMA. E-mail: elifarmabr@gmail.com
} 
ABSTRACT: Chronic diseases are established as an international public health problem, especially in Brazil. Medicinal plants have been widely used to treat such diseases, but without proper care their use can lead to intoxication and even death. This is a descriptive and quantitative field study that aimed to investigate, list and perform the botanical identification of medicinal plants most commonly used by residents of São Luís for the treatment of Hypertension, Diabetes, Asthma and Obesity. The data of the indications were obtained after visits to the main fairs of the city of São Luís. After the survey of the seven plants most indicated for each pathology, they were bought and stored in the form of exsicates for their posthumous botanical identification, to know if they corresponded to what was marketed by the marketers. It was observed that only the part that was attributed to the pharmacological action, was sold, a factor that made botanical identification impossible, raising the discussion about the dangers of the sale of these products. A broad search was made in scientific journals with the popular names of the plants informed by the fairgrounds, in order to verify their effectiveness in the treatment of diseases, being verified that all of them had proven efficacy. The present study provided more information about the consumption habits of medicinal plants of the inhabitants of São Luís, as well as their risks and benefits.

KEYWORDS: Medicinal plants; Chronic diseases; Phytotherapy. 


\section{INTRODUÇÃO}

É sabido que o Brasil detém a maior biodiversidade do mundo, acumulando um total de aproximadamente 55 mil espécies catalogadas. O país também conta com grande arsenal de tradições acerca do potencial terapêutico do uso de plantas medicinais, imortalizado durante os anos pela transmissão entre gerações de tais conhecimentos populares (CARNEIRO, 2014). O SUS (Sistema Único de Saúde) aprovou em 2006 a Política Nacional de Práticas Integrativas e Complementares (PNPIC), o mesmo refere-se a uma política nacional que aconselha a inserção de outras modalidades terapêuticas dentro dos serviços de saúde, a exemplo, o uso de plantas medicinais (BRASIL, 2006).

A ANVISA (Agência Nacional de Vigilância Sanitária) reconhece como medicinal toda planta oriunda de matéria prima ativa vegetal, definido por conhecimento prévio de sua eficácia e dos riscos de seu uso, validado por investigação etno-farmacológica de seu uso, provas tecnocientíficas ou ensaios clínicos com o produto esperado acabado, embalado e rotulado (BRASIL, 2010).

A utilização destes produtos naturais é incentivada principalmente por indicações de amigos e familiares, sendo mínima ou zero a participação de um profissional da área da saúde nesse processo. Tais práticas populares são os principais promotores para a incidência de condutas errôneas, quanto ao uso. Sem os devidos cuidados, seu uso pode acarretar ao paciente graves problemas decorrentes, por exemplo, de intoxicações como resultado de superdosagem, interações com outros fármacos ou alimentos, efeitos colaterais e adversos (VARELAL \& AZEVEDO, 2014).

De igual modo, a forma de fabricação, plantio, coleta e extração detém vital importância no desenvolvimento do produto final. O uso de plantas medicinais requer rigoroso cuidado com o preparo e grande atenção quanto ao controle de qualidade que, se feito de forma incorreta, podem não gerar o efeito desejado e, em situações extremas, desencadear efeito adverso (BADKE et al., 2011).

A utilização de plantas medicinais em conjunto com o uso de outros fármacos para "potencializar" seu efeito sobre as doenças crônicas, assim como no tratamento de outras doenças, tem crescido. Tais medicamentos (ainda que naturais e com baixo poder tóxico) podem levar ao aparecimento de sérios distúrbios, desde que existam fatores de risco, como seu uso concomitantemente com demais medicamentos; podendo levar a um quadro 
totalmente adverso ao desejado: o agravamento no estado homeostático corporal (OLIVEIRA \& MOREIRA, 2010).

As doenças crônicas se estabelecem como um problema de saúde pública, em âmbito internacional, e em especial no Brasil. O ritmo de vida capitalista, cada vez mais corrido, promove o aumento na taxa de sedentários, assim como no número de pessoas que se alimentam de forma inadequada ou que diariamente são expostas a situações de estresse. A prevalência dessas doenças como a Hipertensão, Diabetes, Asma e Obesidade e seus fatores de risco, unidas ao não tratamento adequado (ou pela não manifestação de sintomas, ou pela execução incorreta do tratamento) multiplicam as chances de se ter a ocorrência de complicações severas no organismo humano, como o acidente vascular cerebral (AVC) no caso da Hipertensão, infarto agudo do miocárdio no caso da Diabetes, asfixia no caso da Asma e Esteatose Hepática no caso da Obesidade.

O uso de plantas medicinais sem o aconselhamento médico, sem comprovação científica de sua eficácia e identificação, pode ainda acarretar sérios quadros de intoxicação, neutralização/potencialização (se utilizado simultaneamente com fármacos industrializados) levando ao agravo no quadro clínico do paciente e, em situações extremas, a morte.

Observado tal problema, esse estudo tem por objetivo levantar e identificar as plantas medicinais mais usadas de forma alternativa para tratamento e controle das principais doenças crônicas que acometem a população de São Luís. Além disso, através da identificação botânica, será possível comprovar se as plantas correspondem aos nomes com os quais são comercializadas, possibilitando assim, através da busca em periódicos científicos, a comprovação da sua provável eficácia.

\section{MATERIAL E MÉTODOS}

Trata-se de uma pesquisa de campo, exploratória, com abordagem descritiva e quantitativa. A pesquisa foi realizada no final do segundo semestre de 2016. Inicialmente realizou-se a seleção dos principais pontos de venda de plantas medicinais e fitoterápicos da cidade de São Luís, selecionando-se o Mercado das Tulhas, a Feira da COHAB e a Feira do Mercado Central como pontos de atuação.

Foi realizada uma visita em cada um dos locais citados acima, na qual todos os estabelecimentos de venda de produtos naturais e fitoterápicos foram vistoriados. Os feirantes 
e/ou vendedores foram questionados acerca de quais plantas em seu estabelecimento comercial seriam indicadas para o tratamento da Asma, Diabetes, Hipertensão e Obesidade.

Após as visitas às feiras, os dados coletados foram analisados e uma lista com o nome popular das sete plantas mais indicadas foi feita, onde a primeira planta listada correspondia à planta mais indicada, e assim consecutivamente. Após as sete plantas mais indicadas terem sido encontradas, retornou-se às feiras para a compra das mesmas nos locais que as vendiam pelo menor preço.

As plantas foram armazenadas na forma de exsicatas para a preservação da sua integridade, para sua identificação botânica no Herbário da UFMA; esta identificação revelaria se os nomes populares das plantas ditas pelos feirantes correspondiam às espécies comercializadas. Com base nesses dados, posteriormente realizou-se ampla busca em periódicos científicos sobre a possível eficácia das plantas contra as doenças listadas.

\section{RESULTADOS}

Do total de plantas investigadas $(100 \%)$ nas feiras, obtiveram-se como as mais indicadas vinte e oito $(n=28)$ espécies no total, sendo que para o tratamento da asma $(25 \%)$ foram listadas 7 plantas $(n=7)$, para o tratamento do diabetes $(25 \%)$ foram listadas 7 plantas $(n=7)$, para o tratamento da hipertensão (25\%) foram listadas 7 plantas $(n=7)$ e para o tratamento da obesidade $(25 \%)$ foram listadas também 7 plantas $(n=7)$.

As plantas mais indicadas nas 3 feiras para o tratamento da obesidade, seus nomes populares e a porcentagem de suas respectivas indicações, foram: Baccharis trimera Carqueja (25\%); Maytenus ilicifolia - Espinheira-santa (21\%); Cammelia sinensis - Chá-verde (15,7\%); Equisetum giganteum - Cavalinha (15,3\%); Cyanara scolymus - Alcachofra (9,9\%); Cassia angustifólia - Sena (8,1\%); Hibiscus sabdariffa - Hibisco (5\%). Para o tratamento da hipertensão: Uncaria tomentosa - Unha-de-gato (33\%); Dioclea violacea - Coronha (26,8\%); Equisetum hyemale L. - Cavalinha (13,2\%); Cymbopogoncitratus - Capim-limão (9,5\%); Quassia amara - Pau tenente (6,5\%); Lippia alba N. E. Brown - Erva cidreira (5,8\%); Cuphea carthagenensis - Sete sangrias (5,2\%). Para o tratamento do diabetes: Bahuinia sp - Pata de Vaca (29,2\%); Cissus sicyoides L. - Insulina (22,1\%); Anacardium occidentale L. - Cajueiro (18,3\%); Baccharis trimera DC. - Carqueja (11,7\%); Eucaliptus globulus Labill. - Eucalipto (9,3\%); Caesalpinia ferrea Mart. - Pau-ferro (6,4\%); Urtica spp. - Urtiga (3\%). Para o tratamento da asma: Eucalyptus - Eucalipto (39,9\%); Commiphora leptophloeos - Imburana 
(18,1\%); Cynara cardunculus - Alcachofra (17\%); Chenopodium ambrosioides L.- Mastruz (16,5\%); Urtica spp. - Urtiga (4\%); Vernonia polysphaera - Assa-peixe (3,5\%); Struthanthus flexicalis - Erva Santa (1\%).

\section{DISCUSSÃO}

A comprovação se de fato as plantas comercializadas correspondiam às espécies ditas pelos feirantes não foi possível, pois essas plantas são vendidas em pequenas partes, impossibilitando o processo. Portanto, a listagem das plantas com seus respectivos nomes científicos foi realizada levando-se em consideração o nome popular das plantas, dito pelos feirantes. Realizou-se ampla busca em periódicos científicos e publicações da área de farmacologia e verificou-se que todas as espécies de fato possuem ação terapêutica para as respectivas patologias para as quais são indicadas.

\section{CONCLUSÃO}

A comprovação botânica e científica dessas espécies não foi possível, pois para a sua identificação seria necessária toda a planta ou a maior parte possível, porém, nas feiras, só uma parte das plantas, que é atribuída à eficácia, é vendida. Por não haver comprovação, a confirmação da sua eficácia em periódicos científicos foi feita levando-se em consideração apenas o nome popular das espécies vendidas. Sendo assim, há a necessidade de realizar estudos fitoquímicos para a sua identificação mais específica. No entanto, buscou-se listar os nomes científicos conforme os nomes populares cedidos. Constatou-se também que os feirantes livremente as vendem sem qualquer fiscalização, fato que representa sério risco à saúde dos consumidores. Dessa forma, listaram-se e investigaram-se as plantas mais consumidas pela população, no entanto, não se pode comprovar de fato de que tratava-se das plantas mencionadas, já que apenas obtiveram-se as suas partes e não foi possível saber a origem de onde os feirantes as obtinham. 


\section{REFERÊNCIAS}

BRASIL. Ministério da Saúde. Política Nacional de Plantas Medicinais e Fitoterápicos. Brasília: Ministério da Saúde, 2006. Disponível em

<http://bvsms.saude.gov.br/bvs/publicacoes/politica_nacional_fitoterapicos.pdf>. Acesso em: 18 de março de 2015.

Ministério da Saúde: Agencia Nacional de Vigilância Sanitária. Resolução RDC nº 10, de 09 de março, Brasília, 2010.

BADKE, M. R.; RESSEL, L. B.; BUDÓ, M. L. D.; Plantas medicinais: o saber sustentado na prática do cotidiano popular; Esc. Anna Nery (impr.) 2011 jan/mar; 15 (1):132-139.

BARBOSA, J. H. P.; OLIVEIRA, S. L., SEARA, L. T.; O Papel dos Produtos Finais da Glicação Avançada (AGEs) no Desencadeamento das Complicações Vasculares do Diabetes; Faculdade de Nutrição da Universidade Federal de Alagoas (UFAL), Maceió, AL, Brasil; Arq. Bras. Endocrinologia Metabólica 2008;52/6.

CARNEIRO, F. M.; SILVA, M. J. P.; BORGES, L. L.; Tendências dos estudos com plantas medicinais no Brasil; Revista Sapiência: sociedade, saberes e práticas educacionais UEG/Campus de Iporá, v.3, n. 2, p.44-75 - jul/dez 2014 - ISSN 2238-3565.

MAIA, L. F.; CASTRO, Q. J. T.; RESENDE, F. M. F.; RODRIGUES, R. G.; Plantas medicinais e hipertensão; Artigo Técnico, Centro de Saúde da Universidade Federal de Ouro Preto; Campus Universitário s/n - Ouro Preto/ MG; fev/mar de 2011.

OLIVEIRA, C. J.; MOREIRA, T. M. M; Caracterização do tratamento não-farmacológico de idosos portadores de hipertensão arterial; Rev. Rene. Fortaleza, v. 11, n. 1, p. 76-85, jan./ mar de 2010.

SOCIEDADE BRASILEIRA DE PNEUMOLOGIA E TISIOLOGIA; Diretrizes $\boldsymbol{d a}$ Sociedade Brasileira de Pneumologia e Tisiologia para o Manejo da Asma - 2012; Jornal Bras. Pneumologia. v.38, Suplemento 1, p.S1-S46 Abril 2012. 
SOUSA, A. D. Z.; VARGAS, N. R. C.; CEOLIN, T.; A enfermagem diante da utilização de plantas medicinais no tratamento complementar da hipertensão arterial sistêmica e das dislipidemias; REME - Rev. Min. Enferm.;14(4): 473-478, out./dez., 2010.

SOUZA, C. O.; Influência da inatividade física na ocorrência do sobrepeso e da obesidade em estudantes do ensino fundamental das escolas públicas da cidade do salvador/BA; Universidade Federal da Bahia/Escola de Nutrição, 2008.

VARELAL, D. S.; AZEVEDO, D. M.; Saberes e práticas fitoterápicas de médicos na estratégia saúde da familia. Trab. Educ. Saúde, Rio de Janeiro, v. 12 n. 2, p. 273-290, maio/ago. de 2014. 